\title{
Oral Hypoglycemics in Patients with Type 2 Diabetes and Peripheral Artery Disease
}

Luke Rannelli MD, MSc, Eric Kaplovitch MD, Sonia S. Anand MD, PhD

\section{About the Authors:}

Luke Rannelli MD, MSc, is with the Department of Medicine, Division of General Internal Medicine, University of Calgary, Calgary, Alberta, Canada.

Eric Kaplovitch MD, is with the Department of Medicine, University of Toronto, ON, Canada.

Sonia S. Anand MD PhD is with the Population Health Research Institute and Department of Medicine, McMaster University and Hamilton Health Sciences, ON, Canada.

Corresponding Author: larannel@ucalgary.ca

Submitted: April 18, 2018. Accepted: September 16, 2018. Published: May 21, 2019. DOI:10.22374/cjgim.v14i2.282

\begin{abstract}
Worldwide, in 2010, 202 million people were living with peripheral artery disease (PAD), with a prevalence between $3-12 \%$. The prevalence of PAD is 3 times greater in diabetic patients compared to those with normal glycemia. PAD of the limbs is associated with increased cardiovascular morbidity and mortality, as well as major adverse limb events including acute limb ischemia and amputation. These risks are particularly high in patients who smoke and/or have type 2 diabetes. The goal of treatment in diabetic patients with PAD is to prevent cardiovascular events and prevent further peripheral artery stenosis leading to limb ischemia, and amputation. Poor glycemic control contributes to atherosclerotic progression; however, no randomized control trial evidence exists that demonstrates improved glycemic control reduces the risk of PAD. Oral diabetic medications are designed to lower glucose levels, reduce symptoms and the microvascular complications of diabetes without the inconvenience of daily injections. However, the data supporting the benefit of these medications in diabetic populations with concurrent PAD are limited. We review the evidence for oral hypoglycemic agents in the treatment of patients with concurrent PAD and diabetes.
\end{abstract}

\section{Résumé}

En 2010, 202 millions de personnes dans le monde vivaient avec une maladie artérielle périphérique (MAP), avec une prévalence comprise entre 3 et $12 \%$. La prévalence de la MAP est 3 fois plus élevée chez les patients diabétiques que chez ceux dont la glycémie est normale. La MAP des membres est associée à une augmentation de la morbidité et de la mortalité cardiovasculaires, ainsi quà des événements indésirables majeurs des membres, dont l'ischémie aiguë des membres et l'amputation. Ces risques sont particulièrement élevés chez les patients qui fument et/ou qui souffrent de diabète de type 2. L'objectif du traitement chez les patients diabétiques atteints de MAP est de prévenir les événements cardiovasculaires et de prévenir d’autres sténoses des artères périphériques entraînant une ischémie des membres et l'amputation. Un mauvais contrôle glycémique contribue à la progression de l'athérosclérose ; cependant, il n'existe aucune preuve d’essai clinique comparatif randomisé qui démontre qu'un meilleur contrôle glycémique réduit le 
risque de MAP. Les médicaments oraux pour diabétiques sont conçus pour abaisser la glycémie, réduire les symptômes et les complications microvasculaires du diabète sans les inconvénients des injections quotidiennes. Toutefois, les données à l'appui des bienfaits de ces médicaments chez les populations diabétiques présentant une MAP concomitante sont limitées. Nous examinons les données probantes sur les hypoglycémiants oraux dans le traitement des patients atteints de MAP et de diabète concomitants.

Keywords: hemoglobin Alc, peripheral arterial disease, diabetes, oral hypoglycemic agents

A 57-year-old-female with type 2 diabetes for the last 5-years presented to her primary care physician complaining of pain in her legs with walking, but not at rest. She was a smoker with a body mass index of 21. She had palpable posterior tibial pulses. After being sent for peripheral artery disease (PAD) testing, a new diagnosis of intermittent claudication was made based on her symptoms and low ankle-brachial indices of 0.72 and 0.80 in the right and left legs respectively. The patient was treated with metformin, rosuvastatin, and ramipril and was placed on low dose aspirin following the diagnosis of symptomatic PAD. Smoking cessation and nicotine replacement therapy were offered to the patient. Despite maximal titration of her metformin, her most recent hemoglobin A1c remained elevated at 8.3\%, and her primary care physician was concerned that her sub-optimal hemoglobin A1c may have contributed to her development of $\mathrm{PAD}$, and considered adding a second oral hypoglycemic agent. In light of the results of the EMPA-REG outcome trial and Diabetes Canada Guidelines, a SGLT-2 inhibitor (empagliflozin) was prescribed. ${ }^{1,2}$ The pharmacist filling prescription pointed out that a similar SGLT-2 inhibitor has a United States-FDA warning for increased risk of limb amputation.

\section{What is the Risk of Developing PAD in Patients with Type 2 Diabetes? What is the Impact of PAD on Cardiovascular Health and Risk of Amputation in Diabetic Patients?}

Worldwide, in 2010, 202 million people were living with PAD, with a prevalence between 3 and $12 \% .{ }^{3-5}$ PAD of the lower extremities is usually characterized by flow-limiting artery stenosis resulting in a reduction of blood supply to the limbs. This can manifest as symptoms of intermittent claudication and pain with ambulation in the legs, persistent rest pain, or limb ulceration/gangrene. However, a large percentage of patients remain asymptomatic. PAD of the limbs is associated with increased cardiovascular morbidity and mortality, as well as major adverse limb events including acute limb ischemia and amputation. These risks are particularly high in patients who smoke and/or have type 2 diabetes. ${ }^{6,7}$ Diabetes causes endovascular dysfunction, accelerating atherosclerosis of the vessels. ${ }^{8-10}$
The prevalence of PAD is 3 times greater in diabetic patients compared to those with normal glycemia, ${ }^{9,11}$ and is a common initial presentation of vascular disease in diabetic patients, occurring in $16.2 \%$ of the first manifestation of cardiovascular disease. ${ }^{12}$ The duration and control of a patient's diabetes are associated with an increased risk in the development of PAD. ${ }^{13}$ For example, a $1 \%$ increase in hemoglobin A1c is associated with a $28 \%$ increased risk of PAD development over an 18 year period. ${ }^{13}$ Patients with diabetes and concurrent PAD have a 3-4 fold increase in mortality, ${ }^{14}$ and amputation rates are 5 times higher than non-diabetic patients with PAD. ${ }^{8}$

\section{What is the Impact of Glycemic Control On PAD?}

The goal of treatment in diabetic patients with PAD is to prevent cardiovascular events and prevent further peripheral artery stenosis leading to limb ischemia, and amputation. Poor glycemic control contributes to atherosclerotic progression; however, no randomized control trial evidence exists that demonstrates improved glycemic control reduces the risk of PAD. ${ }^{15}$ A prospective cohort study of 1,637 patients with PAD reported that diabetic patients who were not treated with glucose-lowering medications had a higher mortality and increased rates of peripheral artery interventions compared to patients treated with oral hypoglycemic agents. ${ }^{16}$ Two meta-analyses of the effect of intensive glucose-lowering treatment on mortality, myocardial infarction, stroke, peripheral vascular events (i.e., leg revascularization, PAD or claudication) and amputation, concluded that there was minimal mortality, myocardial infarction or stroke benefit from tight hemoglobin A1c control. ${ }^{17,18}$ However, the 2 meta-analyses did differ in their conclusions on amputations and peripheral vascular events. The initial publication in 2011 did not show an impact of tight glucose control on peripheral vascular events (RR 0.98; $95 \%$ CI: 0.84-1.13) or amputations (RR 0.84; 95 \% CI: 0.54-1.29), ${ }^{17}$ whereas the more recent 2016 publication suggests a reduction in diabetic-related amputations (RR 0.65, 95\% CI: 0.45-0.94). ${ }^{18}$ Of note, the 2016 meta-analysis did not demonstrate a difference between intensive glycemic control and liberal control in peripheral ischemic outcomes (i.e., gangrene, ischemic ulcer, new-onset claudication, new diagnosis of PAD) (RR 0.92 95\% 
CI: 0.67-1.26) ${ }^{18}$ The discrepancy between the 2 meta-analyses conclusion on amputation outcomes may be due to different methodology. The 2016 study included multiple studies that utilized multifactorial interventions, which were excluded from the 2011 meta-analysis. ${ }^{19}$ More recently, in a retrospective cohort study of PAD patients awaiting revascularization (endovascular repair, open surgical repair and hybrid) a lower pre-operative hemoglobin A1c level was associated with a lower risk of adverse limb outcomes/amputations after a 3.5-year follow-up period (i.e., hemoglobin A1c 6.1-7\%; HR 1.03 CI 0.95-1.12; hemoglobin A1c 7.1-8\%; HR 1.15 CI 1.05-1.26; hemoglobin A1c >8\%; 1.27 CI 1.16-1.38) compared to PAD patients without diabetes). ${ }^{20}$ As hyperglycemia contributes to atherosclerosis progression, achievement of intensive glycemic control, defined as a hemoglobin Alc $\leq 7$, is a logical approach in the management of a patient with diabetes and PAD. However, no randomized controlled trials of the effect of tight glycemic control on cardiovascular outcomes and amputation rates have been completed.

\section{Diabetic Agents and PAD}

The majority of patients in the US with diabetes are managed with oral hypoglycemic medications (56.9\%) compared to the use of insulin alone (14\%), or the combination of oral medications and insulin (14.7\%). ${ }^{21}$ Similar prescription use is seen in Canada, where the majority of diabetic patients ( $61 \%)$ between 30 and 90 years of age are prescribed at least one oral hypoglycemic. ${ }^{22}$ Oral diabetic medications are designed to lower blood glucose levels, reduce symptoms and the microvascular complications of diabetes without the inconvenience of daily injections. However, the data supporting benefit of these medications in diabetic populations with concurrent PAD are limited.

The development of metformin, a biguanide has become a mainstay of therapy for patients with type 2 diabetes. Much of the cardiovascular benefit for metformin is derived from the initial UKPDS trial and subsequent 10-year follow-up that demonstrated a mortality and myocardial infractions benefit. ${ }^{23,24}$ However, the impact on mortality and PAD has been controversial. ${ }^{25}$ A meta-analysis of randomized trials among patients with type 2 diabetes examined the impact of metformin on major cardiovascular outcomes, and suggested a non-significant $16 \%$ reduction in all-cause mortality (HR 0.96 95\%: CI 0.84-1.09) and $25 \%$ reduction in myocardial infarction (HR 0.89 95\%: CI $0.75-1.06) .{ }^{25} 2079$ patients were included in the meta-analysis with duration of follow-up ranging from 6 to 212 months, with much of the data driven by the UKPDS data. Subset analyses of diabetic patients on metformin showed a non-significant $19 \%$ reduction in the risk of developing PAD (RR 0.81 95\%: CI $0.50-1.31) .{ }^{23-27}$ The diagnosis of PAD varied widely among the 4 papers included, from a new diagnosis of PAD after angiographic demonstration, to PAD resulting in amputation or death. The 10-year follow-up UKPDS study failed to demonstrate any advantage of metformin to sulphonylureas or insulin in the prevention of PAD in diabetic patients. ${ }^{24}$ To date, there has been no randomized controlled trial data supporting a reduction in cardiovascular death, development of PAD or amputation from PAD in diabetic patients with PAD with metformin.

Sulfonylurea drugs promote insulin secretion through its effect on ATP channels. The UKPDS trial showed microvascular benefit of sulfonylureas and a trend towards macrovascular benefit; however, no data has shown a reduction in PAD in type 2 diabetic patients. ${ }^{28}$ Furthermore, there are concerns about an increased risk of significant hypoglycemic episodes. ${ }^{29-31}$ Therefore the benefit of these medications may not outweigh the risks in the PAD patient population that often have higher rates of cardiovascular disease. ${ }^{29}$ The forthcoming CAROLINA study will study the potential cardiovascular benefits of glimepiride versus linagliptin in the diabetic population. ${ }^{32}$

The effect of thiazolidinediones in improving insulin sensitivity in peripheral tissues made this class of medications an attractive option in patients with high cardiovascular risk. The overall cardiovascular benefit of these medications and possible risk of congestive heart failure in patients with diabetes has been hotly debated. ${ }^{33-35}$ In the PROACTIVE randomized, placebocontrolled trial, treatment with pioglitazone demonstrated a non-significant reduction in the primary outcome of all-cause mortality, myocardial infarction, stroke, amputation and leg revascularization (HR $0.90 \mathrm{CI} 0.80-1.02, \mathrm{P}=0.095) .{ }^{36}$ The secondary composite endpoint of all-cause mortality, non-fatal myocardial infarction, and stroke was significantly reduced compared to placebo (HR 0.84, CI 0.72-0.98, P = 0.027). ${ }^{36}$ However, in the subgroup of diabetic patients with pre-established PAD, pioglitazone did not demonstrate improvement in any major cardiovascular outcomes and was associated with an increase in leg revascularizations (surgical bypass/atherectomy/angioplasty/ thrombolysis) (HR 1.68, CI 1.15-2.47, $\mathrm{P}=0.008$ ). There was no significant increase in lower limb amputations (HR $1.58 \mathrm{CI}$ $0.81-3.12, \mathrm{P}=0.18$ ). The beneficial effects of pioglitazone were only seen in patients without $\mathrm{PAD}$, as the $\mathrm{P}$-values for interaction between $\mathrm{PAD}$ and non-PAD patients were $\mathrm{P}=0.04$ for primary outcome, $\mathrm{P}=0.03$ for acute coronary syndrome, $\mathrm{P}=0.007$ for leg revascularization, and $\mathrm{P}=0.03$ for amputation rates. No other thiazolidinediones class medication have studied diabetic patients in PAD with regards to vascular outcomes.

Animal and observational studies in human populations suggest that the incretin-based oral selective inhibitors of dipeptidyl peptidase 4 (DPP-4) medications may reduce cardiovascular outcomes. ${ }^{37}$ For example, a large population-based observational cohort study from Taiwan of 82,169 patients started on DPP-4 
medication demonstrated a $16 \%$ decrease in the risk of developing PAD and a 35\% reduced risk of amputation. ${ }^{37}$ However, these findings have not been confirmed in randomized trials of DPP-4 inhibitors as no reduction in the major cardiovascular events (MACE) outcome or PAD outcomes have been observed. ${ }^{31}$

In contrast, the incretin modulator GLP-1 agonists have demonstrated a significant reduction in cardiovascular events in both the large LEADER trial and SUSTAIN-6 trial. ${ }^{35,38,39}$ Liraglutide an injectable agent reduced the risk of cardiovascular death, myocardial infarction and stroke by $13 \%$ in patients with a baseline elevated cardiovascular risk. ${ }^{38} \mathrm{~A}$ similar trend was observed in semaglutide, with a $26 \%$ reduced in combined primary outcome of cardiovascular death, non-fatal myocardial infarction and nonfatal stroke. ${ }^{39}$ A significant reduction in nephropathy has also been demonstrated with both liraglutide and semaglutide. ${ }^{38,39}$ Though the mechanism remains unclear, the weight, blood pressure and lipid reductions with GLP-1 inhibitors may explain the improvement in cardiovascular events but this remains unclear. However, the effect of GLP-1 agonists on PAD development, progression, revascularization procedure rates and amputations secondary to PAD have not been well studied.

The newest and most promising class of hypoglycemic agents are the SGLT-2 inhibitors, which along with liraglutide, are recommended as a second-line agent for patients with documented clinical cardiovascular disease. ${ }^{1}$ SGLT-2 inhibitors block sodium-glucose cotransport in the renal tubules, increasing urinary glucose exertion. The CDA recommendation came primarily from the EMPA-REG OUTCOME trial in which the novel SGLT-2 inhibitor, empagliflozin, demonstrated a $14 \%$ (HR 0.86; 95\% CI 0.74-0.99) relative risk reduction in MACE and a $32 \%$ (HR 0.68 95\% CI 0.57-0.82) reduction in all-cause mortality ${ }^{2,40}$ among the 7,020 patients randomized. In addition, empagliflozin slowed progression in kidney disease by $6 \%$ and risk of macroalbuminuria by $5 \%$ (nephropathy HR $0.61 \mathrm{CI}$ 0.53-0.70; macroalbuminuria HR $0.6295 \%$ CI $0.54-0.72){ }^{2}$ In a recent subgroup analysis of 1461 patients with PAD included in the EMPA-REG OUTCOME trial, empagliflozin demonstrated a $38 \%$ reduction in all-cause mortality (HR $0.6295 \%$ CI $0.44-0.88$ ) and a $43 \%$ reduction in cardiovascular deaths (HR 0.57 95\% CI $0.37-0.88$ ) with no increased rate of limb amputations (HR 0.84 95\% CI 0.54-1.32). ${ }^{41}$ The other SGLT-2 inhibitor approved in Canada is canagliflozin, which in the CANVAS trial, demonstrated similar reductions in MACE (HR 0.86 95\% CI 0.75-0.97). ${ }^{40}$ However, in contrast to empagliflozin, an unexpected two-fold increase in the risk of lower limb amputations was observed with canagliflozin, with the majority (71\% - HR $1.9795 \%$ CI 1.41-2.75) of amputations occurring at the toe or metatarsal level. ${ }^{40,42}$ The highest risk of amputation occurred among patients with previous amputation or PAD and tended to occur later in the trial (1.5 years) after drug initiation. This observation underlies canagliflozin's black box warning from the US FDA for amputation risk. ${ }^{42}$ The exact mechanism by which amputations are increased remains unclear. In unpublished subgroup data on etiologies of amputations in patients on canagliflozin, acute limb ischemia, a precursor to amputation, was also increased by two-fold increase compared to placebo ( $12.9 \%$ canagliflozin vs. $6.4 \%$ placebo). ${ }^{43}$ To date there has been no reported increased risk of amputation rates in other SGLT-2 inhibitors thus far (dapagliflozin or empagliflozin). ${ }^{42,44}$ Furthermore the prevalence of PAD (20\%) between CANVAS and EMPA-REG OUTCOME were similar, suggesting that clinical characteristics of the studies do not explain the increased amputation rates. ${ }^{42}$ Recently, an observational cohort study conducted by the pharmaceutical company responsible for canagliflozin, did not demonstrate an increased risk of amputation in 63,845 new users of canagliflozin with and without established cardiovascular disease $(20 \%$ and $8 \%$ of whom had PAD). ${ }^{44}$ Further randomized clinical studies with canagliflozin and its impact of amputation rates and PAD are required. It should be noted that since this report, the DECLARE trial with dapagliflozin is now required by the European Medicines Agency to report amputation events. ${ }^{45}$

\section{Case Revisited}

After a discussion regarding risks and benefits and importance of lifestyle changes (smoking cessation and regular walking, and foot care), the primary care physician prescribed empagliflozin at 10-mg daily given its cardiovascular benefit and reduction in all-cause mortality. While it would also have been reasonable to recommend liraglutide, empagliflozin's benefit in reducing nephropathy and renal impairment may make it the preferred agent in patients at risk or with documented kidney dysfunction. We now have second line hypoglycemic agents with proven benefit to diabetic patients with PAD.

\section{References}

1. Committee CDACPGE. Pharmacologic management of type 2 diabetes: 2016 interim update. Can J Diabetes 2016;40(6):484-6.

2. Zinman B, Wanner C, Lachin JM, et al. Empagliflozin, cardiovascular outcomes, and mortality in type 2 diabetes. N Engl J Med 2015;373(22):2117-28.

3. Gerhard-Herman MD, Gornik HL, Barrett C, et al. 2016 AHA/ACC Guideline on the Management of Patients with Lower Extremity Peripheral Artery Disease: Executive Summary. Vasc Med 2017;22(3):NP1-NP43.

4. Novo S. Classification, epidemiology, risk factors, and natural history of peripheral arterial disease. Diabetes Obes Metab 2002;4 Suppl 2:S1-6.

5. Fowkes FG, Rudan D, Rudan I, et al. Comparison of global estimates of prevalence and risk factors for peripheral artery disease in 2000 and 2010: a systematic review and analysis. Lancet 2013;382(9901):1329-40.

6. Jones WS, Patel MR, Rockman CB, et al. Association of the ankle-brachial index with history of myocardial infarction and stroke. Am Heart J 2014;167(4):499-505. 
7. Emdin CA, Anderson SG, Callender T, et al. Usual blood pressure, peripheral arterial disease, and vascular risk: cohort study of 4.2 million adults. BMJ 2015;351:h4865.

8. Jude EB, Oyibo SO, Chalmers N, Boulton AJ. Peripheral arterial disease in diabetic and nondiabetic patients: a comparison of severity and outcome. Diabetes Care 2001;24(8):1433-7.

9. Jude EB, Eleftheriadou I, Tentolouris N. Peripheral arterial disease in diabetes--a review. Diabet Med 2010;27(1):4-14.

10. Marso SP, Hiatt WR. Peripheral arterial disease in patients with diabetes. J Am Coll Cardiol 2006;47(5):921-9.

11. Selvin E, Erlinger TP. Prevalence of and risk factors for peripheral arterial disease in the United States: results from the National Health and Nutrition Examination Survey, 1999-2000. Circulation 2004;110(6):738-43.

12. Shah AD, Langenberg C, Rapsomaniki E, et al. Type 2 diabetes and incidence of cardiovascular diseases: a cohort study in 1.9 million people. Lancet Diabetes Endocrinol 2015;3(2):105-13.

13. Adler AI, Stevens RJ, Neil A, et al. UKPDS 59: hyperglycemia and other potentially modifiable risk factors for peripheral vascular disease in type 2 diabetes. Diabetes Care 2002;25(5):894-9.

14. Beks PJ, Mackaay AJ, de Neeling JN, et al. Peripheral arterial disease in relation to glycaemic level in an elderly Caucasian population: the Hoorn study. Diabetologia 1995;38(1):86-96.

15. Patel A, MacMahon S, Chalmers J, et al. Intensive blood glucose control and vascular outcomes in patients with type 2 diabetes. $\mathrm{N}$ Engl J Med 2008;358(24):2560-72.

16. Golledge J, Quigley F, Velu R, Walker PJ, Moxon JV. Association of impaired fasting glucose, diabetes and their management with the presentation and outcome of peripheral artery disease: a cohort study. Cardiovasc Diabetol. 2014; 13:147.

17. Boussageon R, Bejan-Angoulvant T, Saadatian-Elahi M, Lafont $S$, Bergeonneau C, Kassaï B, et al. Effect of intensive glucose lowering treatment on all cause mortality, cardiovascular death, and microvascular events in type 2 diabetes: meta-analysis of randomised controlled trials. BMJ 2011;343:d4169.

18. Hasan R, Firwana B, Elraiyah T, et al. A systematic review and meta-analysis of glycemic control for the prevention of diabetic foot syndrome. J Vasc Surg 2016;63(2 Suppl):22S-8S.e1-2.

19. Rehman MB, Tudrej BV, Boussageon R. Regarding "A systematic review and meta-analysis of glycemic control for the prevention of diabetic foot syndrome". J Vasc Surg 2016;64(1):264-5.

20. Arya S, Binney ZO, Khakharia A, et al. High hemoglobin Alc associated with increased adverse limb events in peripheral arterial disease patients undergoing revascularization. J Vasc Surg 2017.

21. Prevention CfDCa. National Diabetes Statistics Report: Estimates of Diabetes and Its Burden in the United States Atlanta, GA: U.S. Department of Health and Human Services; 2014. Available at: https://stacks.cdc.gov/view/ cdc/23442/cdc_23442_DS1.pdf?

22. 22. Canada PHA. Proportion and number of diabetes medications $\dagger$ among individuals aged 12 years and older with self-reported diabetes, by age group and medication type, Canada, 2009-2010: Government of Canada; 2011. Available at: https://www.canada.ca/en/public-health/services/chronicdiseases/reports-publications/diabetes/diabetes-canada-facts-figures-apublic-health-perspective/chapter-2.html

23. UK Prospective Diabetes Study (UKPDS) Group. Effect of intensive bloodglucose control with metformin on complications in overweight patients with type 2 diabetes (UKPDS 34). Lancet 1998;352(9131):854-65.

24. Holman RR, Paul SK, Bethel MA, et al. 10-year follow-up of intensive glucose control in type 2 diabetes. N Engl J Med 2008;359(15):1577-89.

25. Griffin SJ, Leaver JK, Irving GJ. Impact of metformin on cardiovascular disease: a meta-analysis of randomised trials among people with type 2 diabetes. Diabetologia 2017.
26. Gram J, Henriksen JE, Grodum E, et al. Pharmacological treatment of the pathogenetic defects in type 2 diabetes: the randomized multicenter South Danish Diabetes Study. Diabet Care 2011;34(1):27-33.

27. Kooy A, de Jager J, Lehert P, et al. Long-term effects of metformin on metabolism and microvascular and macrovascular disease in patients with type 2 diabetes mellitus. Arch Intern Med 2009;169(6):616-25.

28. UK Prospective Diabetes Study (UKPDS) Group. Intensive blood-glucose control with sulphonylureas or insulin compared with conventional treatment and risk of complications in patients with type 2 diabetes (UKPDS 33). Lancet 1998;352(9131):837-53.

29. Thulé PM, Umpierrez G. Sulfonylureas: a new look at old therapy. Curr Diab Rep 2014;14(4):473.

30. Morgan CL, Poole CD, Evans M, et al. What next after metformin? A retrospective evaluation of the outcome of second-line, glucose-lowering therapies in people with type 2 diabetes. J Clin Endocrinol Metab 2012;97(12):4605-12.

31. Lathief S, Inzucchi SE. Approach to diabetes management in patients with CVD. Trends Cardiovasc Med 2016;26(2):165-79.

32. Marx N, Rosenstock J, Kahn SE, et al. Design and baseline characteristics of the CARdiovascular Outcome Trial of LINAgliptin Versus Glimepiride in Type 2 Diabetes (CAROLINA ${ }^{\star}$ ). Diab Vasc Dis Res 2015;12(3):164-74.

33. Singh S, Bhat J, Wang PH. Cardiovascular effects of anti-diabetic medications in type 2 diabetes mellitus. Curr Cardiol Rep 2013;15(1):327.

34. Rohatgi A, McGuire DK. Effects of the thiazolidinedione medications on micro- and macrovascular complications in patients with diabetes--update 2008. Cardiovasc Drugs Ther 2008;22(3):233-40.

35. Nauck MA, Meier JJ, Cavender MA, et al. Cardiovascular actions and clinical outcomes with glucagon-like peptide-1 receptor agonists and dipeptidyl peptidase-4 inhibitors. Circulation 2017;136(9):849-70.

36. Dormandy JA, Betteridge DJ, Schernthaner G, et al. Impact of peripheral arterial disease in patients with diabetes--results from PROactive (PROactive 11). Atherosclerosis. 2009;202(1):272-81.

37. Chang CC, Chen YT, Hsu CY, et al. Dipeptidyl peptidase-4 inhibitors, peripheral arterial disease, and lower extremity amputation risk in diabetic patients. Am J Med 2017;130(3):348-55.

38. Marso SP, Daniels GH, Brown-Frandsen K, et al. Liraglutide and cardiovascular outcomes in type 2 diabetes. $\mathrm{N}$ Engl J Med 2016;375(4):311-22.

39. Marso SP, Bain SC, Consoli A, et al. Semaglutide and cardiovascular outcomes in patients with type 2 diabetes. N Engl J Med 2016;375(19):1834-44.

40. Neal B, Perkovic V, Mahaffey KW, et al. Canagliflozin and cardiovascular and renal events in type 2 diabetes. N Engl J Med 2017;377(7):644-57.

41. Verma S, Mazer CD, Al-Omran M, et al. Cardiovascular outcomes and safety of empagliflozin in patients with type 2 diabetes mellitus and peripheral artery disease: a subanalysis of EMPA-REG OUTCOME. Circulation 2017.

42. Fadini GP, Avogaro A. SGTL2 inhibitors and amputations in the US FDA Adverse Event Reporting System. Lancet Diabet Endocrin 2017;5(9):680-1.

43. Matthews D, Fulcher G, deZeeuw D, Percovic V, Neal B, Ferrannin iE. Program and abstracts of the 53rd Annual Meeting of the European Association for the Study of Diabetes; September 11-15, 2017; Lisbon, Portugal. 2017. Available at: http://www.georgeinstitute.org/media-releases/ major-study-heralds-new-era-in-treatment-of-type-2-diabetes-canvasresults-available.

44. Yuan Z, DeFalco FJ, Ryan PB, et al. Risk of lower extremity amputations in people with type 2 diabetes mellitus treated with sodium-glucose cotransporter-2 inhibitors in the USA: A retrospective cohort study. Diabetes Obes Metab 2017.

45. Raz I, Wiviott S. Multicenter Trial to Evaluate the Effect of Dapagliflozin on the Incidence of Cardiovascular Events (DECLARE-TIMI58) 2017. Available at: https://clinicaltrials.gov/ct2/show/NCT01730534. 